\title{
Estabilidad, sensibilidad y antropización de los paisajes de la Reserva de la Biosfera Buenavista, Cuba
}

\author{
Stability, sensitivity and antropisation of landscapes of \\ Buenavista Biosphere Reserve, Cuba
}

\author{
Julio César García-Espino ${ }^{1}$ \\ Carlos Lázaro Valdés Fernández ${ }^{2}$ \\ Universidad de La Habana, Cuba
}

\begin{abstract}
Resumen
La concepción teórico-metodológica empleada en este artículo se fundamenta en la geoecología, disciplina que emplea la concepción sistémica en el estudio de los paisajes. Concebidos, así como un sistema autorregulado, complejo y abierto, en la medida en que experimenta el impacto antropogénico. El trabajo tiene como objetivo realizar el análisis de las diferentes unidades de paisajes de la Reserva de la biosfera Buenavista, con el fin de establecer su estado en cuanto a la estabilidad, la sensibilidad y el grado de antropización. Como primer paso fueron inventariadas y cartografiadas las unidades de paisaje presentes en el área de estudio. Posteriormente, se establecieron los distintos grados de estabilidad natural y tecnogénica, cuyo análisis conjunto permitió determinar los niveles de sensibilidad de los paisajes. Finalmente, se determinan las escalas de naturalidad de las distintas unidades de paisaje, mediante el uso de los índices naturalidad y sinantropismo.
\end{abstract}

Palabras clave: geoecología, paisajes, estabilidad, sensibilidad, naturalidad.

\begin{abstract}
The theoretical-methodological conception used in this article is based on geoecology, a discipline that uses the systemic conception in the study of landscapes, conceived as well as a self-regulated, complex and open system, insofar as it experiences the anthropogenic impact. The objective of this work is to analyze the different landscape units of the Buenavista Biosphere Reserve, in order to

1 Máster en Geografía, Facultad de Geografía, Universidad de La Habana. Calle L \# 353 piso 6 e/ 23 y 21, Plaza de la Revolución, La Habana, Cuba. imperator@geo.uh.cu

2 Licenciado en Geografía, Facultad de Geografía, Universidad de La Habana. Calle L \# 353 piso 6 e/ 23 y 21, Plaza de la Revolución, La Habana,Cuba. carlos.valdes@geo.uh.cu
\end{abstract}


establish their status regarding stability, sensitivity and the degree of anthropization. As a principal step, the landscape units present in the study area were inventoried and mapped. Later, different degrees of natural and technogenic stability are established, whose joint analysis allowed to determine the levels of sensitivity or fragility of landscapes. Finally, the naturalness scales of the different landscape units are identified, using the indices of naturalness and synanthropism.

Keywords: geoecology, landscapes, stability, sensitivity, naturalness.

\section{Introducción}

El deterioro ambiental es uno de los efectos directos ocasionados por el uso irracional de los recursos naturales. Este se entiende como la pérdida de la calidad ambiental, a consecuencia de la actividad del hombre sobre el ambiente, por lo que puede considerarse como una respuesta al uso ineficiente de los bienes y servicios ambientales por la sociedad (Acevedo, 2014). De manera que los estudios destinados a prevenir, reducir y finalmente, eliminar los impactos negativos al medio físico causados por las actividades humanas, constituyen una herramienta necesaria en la compleja tarea de la organización, gestión y conservación ambiental, con el fin de lograr la utilización racional de sus recursos y fomentar el desarrollo según sus potencialidades y limitantes.

Un paso importante en la promoción de la conservación y el desarrollo de los espacios naturales fue la creación del Programa el Hombre y la Biosfera (MAB) de la UNESCO. El MAB establece la creación de las reservas de biosfera como zonas que pueden contener ecosistemas terrestres, costeros y/o marinos, representativos de su región biogeográfica con importancia para la conservación de la biodiversidad. Cada reserva de biosfera combina tres funciones básicas interconectadas: la conservación, el desarrollo y el apoyo logístico (UNESCO, 2015). En Cuba existen actualmente seis reservas de la biosfera, las cuales forman parte del Sistema Nacional de Áreas Protegidas (SNAP) como áreas de significación nacional.

En el caso específico de la Reserva de la biosfera Buenavista, desde 2010 posee la categoría de manejo de Área Protegida de Recursos Manejados (APRM). Las áreas protegidas bajo esta categoría son áreas operadas principalmente para la utilización sostenible de los ecosistemas naturales, en las cuales deben conjugarse de manera armónica la protección de la diversidad biológica y otros valores y el desarrollo regional y nacional. 
Sin embargo, la Reserva de la biosfera Buenavista ha sido objeto de numerosas e importantes acciones, principalmente de origen antrópico, que han provocado su modificación. El territorio ha transitado por varias etapas de asimilación, en las cuales sobresalen la actividad agrícola, forestal, urbana y recientemente, la actividad turística, con un considerable desarrollo en la construcción de infraestructura hotelera, principalmente en la zona de los cayos.

Según Burgui (2010), la aplicación de un modelo de desarrollo sobredimensionado en la zona de la cayería norte, basado fundamentalmente en la tendencia de sol y playa, ha ocasionado efectos adversos para el medio ambiente en esas áreas. Entre las consecuencias negativas se cuenta, principalmente, la fragmentación de las unidades de paisaje y la pérdida de hábitat natural, lo que se une a otros factores igualmente preocupantes como la contaminación, la pérdida de calidad estética y la introducción de especies exóticas.

Teniendo en cuenta la problemática abordada anteriormente, se entiende la necesidad de llevar a cabo estudios que aporten información dirigida a esclarecer las propiedades y el estado de las distintas unidades de paisaje en la Reserva de la biosfera Buenavista. Estos estudios pueden ser útiles, con el fin de facilitar y servir de base en la toma de decisiones de carácter espacial y de contribuir a una adecuada gestión ambiental. Desde el punto de vista teórico, llevar a cabo trabajos similares a este constituye una muestra de la aplicación de la Geoecología de los paisajes en el campo medioambiental y en el ámbito de las áreas protegidas, al estar relacionado con la propuesta de proyectar las redes de áreas protegidas basadas en el paisaje como unidad espacial de referencia.

\section{Área de estudio}

Buenavista abarca una superficie de $3196,38 \mathrm{~km}^{2}$ entre áreas terrestres y marinas, perteneciente a la región marino-costera del centro-norte de Cuba. Forma parte del archipiélago Sabana-Camagüey y ocupa áreas en los municipios Yaguajay, Caibarién y Chambas, pertenecientes a las provincias Sancti Spíritus, Villa Clara y Ciego de Ávila, respectivamente (figura 1). Se encuentra entre las coordenadas $22^{\circ} 46^{\prime} \mathrm{N}$; $78^{\circ} 53^{\prime} \mathrm{W}$ por el norte y $22^{\circ} 08^{\prime} \mathrm{N}$; $78^{\circ} 46^{\prime} \mathrm{W}$ por el sur. 
Dentro del área de estudio se incluyen nueve áreas núcleos con diferentes categorías de manejo: Parque nacional (Caguanes y Los Caimanes), Refugios de fauna (Las Loras y Cayo Santa María), Elemento natural destacado (Dunas de Pilar, Boquerón, La Chucha y Loma La Tasajera), la Reserva ecológica Cayo Francés y el APRM Jobo Rosado. Estas áreas núcleos son ampliamente diversas e incluyen: manglares, arrecifes coralinos, zonas de dunas activas y herbazal de ciénagas. El área funciona como reservorio de variadas especies, sobresale la fauna, tanto residente, como migratoria, procedente de la región biogeográfica del Neártico, que comprende a la mayor parte de América del Norte (incluyendo a Groenlandia) y las montañas de México. En el año 1989, la Comisión Nacional de Patrimonio otorgó a 13 sitios arqueológicos dentro de la Reserva la categoría de Monumento Local; 12 de estos son cuevas con pictografías aborígenes.

Figura 1. Ubicación del área de estudio

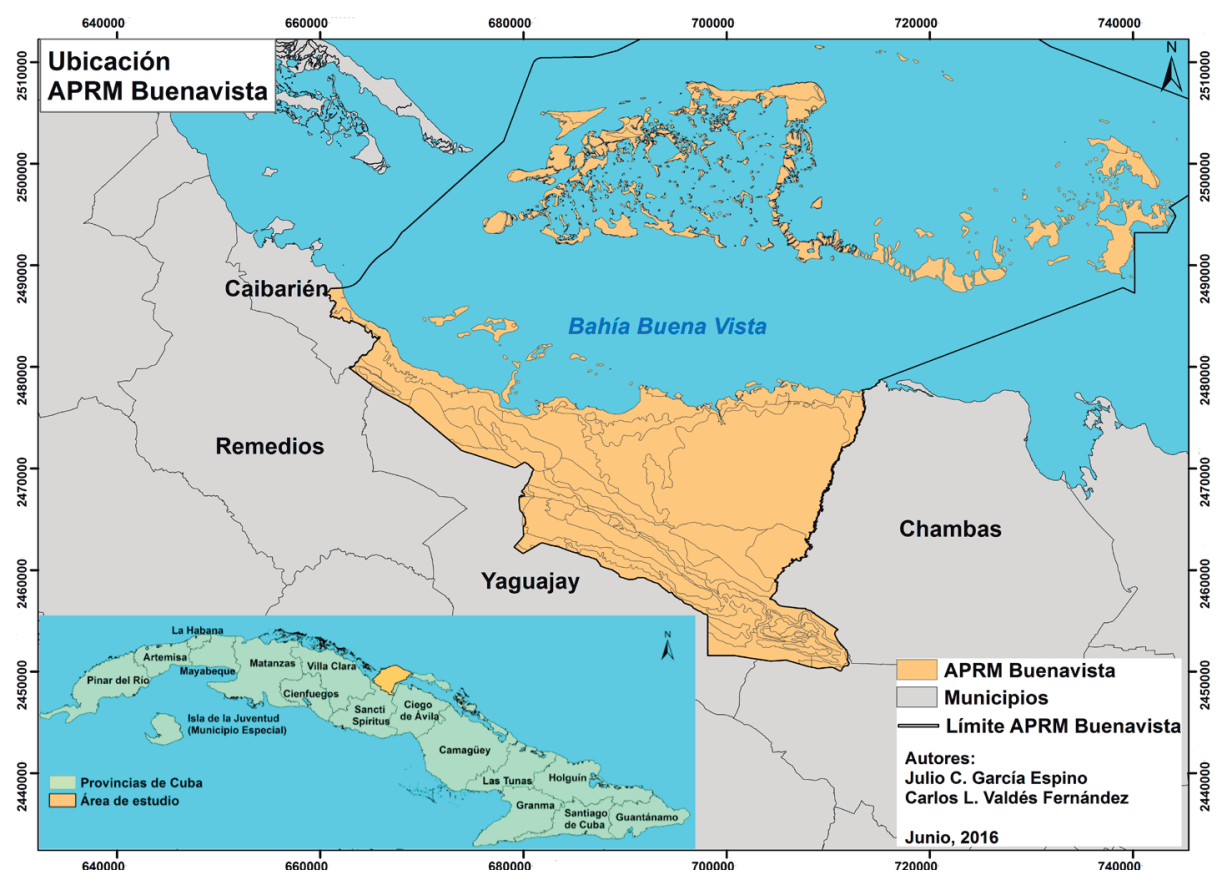

Fuente: Elaborado por los autores a partir de las bases cartográficas de GEOCUBA, 2016 


\section{Metodología}

La concepción teórico-metodológica de este trabajo se fundamenta en la Geoecología de los paisajes, disciplina científica que incorpora una visión mediante la cual se transita del estudio de los objetos fragmentados al análisis de la totalidad sistémica, la cual concibe al paisaje como un sistema espacial, compuesto por elementos naturales y antrópicos, que modifican o transforman las propiedades de los paisajes naturales originales, las cuales difieren en dependencia del grado de naturalidad y de antropización (Mateo, 2011).

La metodología usada tiene como fin el cálculo de los indicadores geoecológicos sintéticos seleccionados y su posterior análisis y representación cartográfica. De manera que estos puedan ofrecer información sobre los distintos procesos de modificación que presenta el área de estudio y su diferenciación espacial.

El desarrollo de la metodología consiste en el inventario y la cartografía de las unidades de paisaje de la Reserva de la biosfera Buenavista como primer paso para el posterior cálculo de la estabilidad, sensibilidad y la naturalidad que estas presentan; como elementos integradores de sus propiedades principales, de sus características estructuro-funcionales y de la influencia antrópica. La obtención, el análisis y la representación espacial de los resultados completan el diseño metodológico del estudio.

\section{Estabilidad del paisaje}

La estabilidad es la capacidad de conservar la estructura y las propiedades de los paisajes, propiedad natural fundamental de estos, expresando el grado de posibilidad del funcionamiento de los geocomplejos, de forma tal que se garantice la reproducción de sus recursos y de otras funciones vitales (Mateo, 2000 y Acevedo, 1996). Con el objetivo de facilitar su análisis se pueden distinguir la estabilidad natural y la tecnogénica, en cuya determinación se utilizan métodos matriciales cualitativos, los cuales se basan en la selección de factores desestabilizadores de los paisajes que influyen en las capacidades actuales de los mismos para responder ante determinados impactos.

La estabilidad natural (EN) se entiende como la capacidad que poseen las diferentes unidades de paisaje para responder ante la acción de los 
agentes y procesos de tipo natural (Sigarreta, 2000). Para el cálculo de la misma se procede a la determinación de la estabilidad interna y de la estabilidad externa, a partir de la evaluación de los factores de peligros internos y externos, respectivamente; según la metodología propuesta por Díaz (1997) en Mateo (2011), en la cual, la estabilidad natural vendría dada por la suma de la interna y la externa.

Luego del cálculo de los valores de la estabilidad interna y externa, se establecieron los siguientes rangos para la EN:

$$
\begin{aligned}
& \text { EN }<3 \text { Estable } \\
& \text { EN 3- } 4 \text { Medianamente estable } \\
& \text { EN }>4 \text { Inestable }
\end{aligned}
$$

En las investigaciones de los autores consultados (Lemes, 2000; García, 2002; Venegas, 2004; Sánchez, 2005; Fajardo, 2008; Aguilar, 2009; Ramón, Salinas y Lorenzo, 2013; Calvo, 2013; Cruañas, 2013;), se mencionan los factores de peligro internos y externos tomados en cuenta para el cálculo de la estabilidad natural; sin embargo, el método para la evaluación de los mismos no queda definida en la metodología. Para la evaluación de los factores de peligro internos y externos se tomaron una serie de indicadores, estableciendo para cada uno diferentes rangos, que van desde uno hasta tres, donde uno representa los valores más favorables para la estabilidad y tres los más desfavorables.

Los factores de peligro internos tomados en cuenta fueron la capacidad protectora de la cubierta vegetal, el grado de inclinación de la pendiente, la erosión potencial y el grado de compactación del sustrato rocoso. Mientras que los factores de peligro externos evaluados fueron la incidencia de huracanes tropicales, los sismos, las sequías y las inundaciones.

Para la evaluación de estos factores fue necesario el uso de una serie de materiales que permitieron determinar el comportamiento favorable o no de cada uno de ellos en el área de estudio. Estos materiales son el mapa de vegetación natural y seminatural de Cuba (Estrada, et al., 2013), el mapa de zonación sísmica con fines de ingeniería (Chuy, 2001), la Cronología de los huracanes de Cuba confeccionada por el Instituto de Meteorología (INSMET) del Ministerio de Ciencia, Tecnología y Medio Ambiente (CITMA), las bases de datos del Centro Nacional de Huracanes de La 
Florida (2014); así como los mapas de peligro integrado por sequía agrícola y meteorológica y de susceptibilidad ante inundaciones por fuertes lluvias de las provincias Villa Clara, Sancti Spíritus y Ciego de Ávila, confeccionados por el grupo de Peligro Vulnerabilidad y Riesgo de la Agencia de Medio Ambiente perteneciente al CITMA.

La estabilidad tecnogénica (ET) se entiende como la capacidad de respuesta del paisaje ante el conjunto de acciones antrópicas. Según Acevedo (1996), se evalúa cualitativamente, teniendo en cuenta las afectaciones por el impacto antrópico y los procesos físico-geográficos actuantes.

Para el cálculo de esta, se establece un cociente entre el porciento de área que ha sufrido modificación antrópica en las unidades (MA) y el porcentaje de modificación que se considera aceptable (MAP). Este último se adopta de forma diferenciada para cada unidad, asumiéndose de estudios anteriores (Sigarreta, 2000; Lemes, 2000), donde las que tienen estabilidad natural baja requieren de un $90 \%$ de naturalidad, las de estabilidad media un $75 \%$ y las de estabilidad alta un $60 \%$ de naturalidad. De esta forma la fórmula para su cálculo es la siguiente: ET $=$ \% MA / \% MAP. Como modificación antrópica se tomaron las áreas de pastos y cultivos, las zonas urbanizadas, los embalses y las zonas con infraestructura productiva.

$$
\begin{aligned}
& \text { ET }<0,5 \text { Estable } \\
& \text { ET 0,5- } 1 \text { Medianamente estable } \\
& \text { ET }>1 \text { Inestable }
\end{aligned}
$$

\section{Sensibilidad del paisaje}

Esta puede caracterizarse según la combinación del comportamiento de la estabilidad natural, y la estabilidad tecnógenica (Acevedo, 1996; Del Risco, 2000 y Sigarreta, 2000, en Aguilar, 2009). Por lo que, para su determinación, se procede por medio de una matriz de análisis de la sensibilidad (tabla 1), que combina el comportamiento de ambos, partiendo de la idea de que a mayor grado de inestabilidad natural y de inestabilidad tecnogénica, los paisajes deben ser más sensibles. 
Julio César Garcia-Espino - Carlos Lázaro Valdés Fernández Stability, sensitivity and antropisation of landscapes of Buenavista Biosphere Reserve, Cuba

Tabla 1. Matriz de análisis de la sensibilidad

\begin{tabular}{|c|c|c|c|}
\hline \multirow{2}{*}{$\begin{array}{c}\text { Estabilidad } \\
\text { Natural }\end{array}$} & Estable & Medianamente estable & Inestable \\
\cline { 2 - 4 } Estable & Muy poco sensibles & Poco sensibles & $\begin{array}{c}\text { Moderadamente } \\
\text { Sensibles }\end{array}$ \\
\hline $\begin{array}{c}\text { Medianamente } \\
\text { estable }\end{array}$ & Poco sensibles & Moderadamente sensibles & Sensibles \\
\hline Inestable & Moderadamente sensibles & Sensibles & Muy sensibles \\
\hline
\end{tabular}

Fuente: Tomado de Lemes, 2000.

\section{Antropización de los paisajes}

El estudio de la antropización de los paisajes se dirige en lo fundamental a la determinación y el análisis de los problemas referentes a la modificación y transformación de los mismos, debido a causas de origen antrópico.

Con el fin de estudiar la antropización de las distintas unidades de paisajes del área de estudio, se utilizaron los resultados del indicador naturalidad. A los resultados de este indicador se llega a través de la combinación entre la clasificación de los paisajes, según la escala de naturalidad y el índice de sinantropismo.

Entre los aspectos para tener en cuenta para la escala de naturalidad se encuentran los elementos bióticos -naturales o de origen antrópico- y su presencia o ausencia. Los elementos artificiales, que incluyen instalaciones construidas, contaminantes. También se valora la entrada de energía y materia, entiéndase las actividades antrópicas que conllevan a transformaciones. Las alteraciones físicas, principalmente en la geomorfología y la topografía. Otro aspecto es la extracción de elementos, tanto bióticos como abióticos - caza, pesca, cosechas; minería, extracción acuífera-. Se examina, además, el nivel de fragmentación de la cobertura natural debido a vías de comunicación, actividades económicas, etc. Como último aspecto se evalúa la dinámica de las unidades de paisaje, teniendo en cuenta su funcionamiento natural y su manipulación, control y dominio por el hombre.

Estos aspectos se concretan en siete criterios diagnósticos y se evalúan usando un rango de valores del 1 -condiciones paupérrimas- al 10 -condiciones óptimas-. Para cada comarca se promedian los valores de los siete criterios y la cifra resultante indica el lugar en la escala de naturalidad.

En cuanto al cálculo del índice de sinantropismo, el primer paso es darle a cada especie registrada dentro del área la categoría sinantrópica, 
usando la clasificación de Ricardo, Pouyú y Herrera (1990). Las categorías para calcular el índice de sinantropismo son: apófitas (nativas, indígenas), antropófitas (introducidas, de forma intencional o no), y parapófitas (no se tiene información de su lugar de origen).

Debido a la ausencia de inventarios florísticos para cada unidad de paisaje, se tomó como alternativa, generalizar las listas florísticas confeccionadas con otros fines en diferentes zonas dentro del área de estudio, las cuales fueron tomadas como áreas de referencia dentro de dichas unidades de paisajes.

Después de tener clasificadas todas las especies del área de estudio dentro de las tres categorías de sinantropismo mencionadas, se lleva a cabo el cálculo mediante la fórmula:

$$
\begin{aligned}
& \text { Is }=(\mathrm{N} 1-\mathrm{N} 2) /(\mathrm{N}-\mathrm{N} 3) \\
& \text { En la que: }
\end{aligned}
$$

Is: índice de sinantropismo

N1: Número de especies nativas

N2: Número de especies introducidas

N3: Número de especies desconocidas

$\mathbf{N}$ : Total de especies encontradas en el área

Los valores resultantes se ubican en los rangos de correlación cualitativa y de esta manera, queda asignado un valor cuantitativo para cada clase (tabla 2).

Tabla 2. Clave interpretativa para la clasificación cualitativa, según los resultados de la aplicación del índice de sinantropismo

\begin{tabular}{|l|c|}
\hline \multicolumn{1}{|c|}{ Clave } & Valor asignado \\
\hline $\begin{array}{l}\text { Is }>0.5 \text { Indica que el ecosistema está en buen estado de } \\
\text { conservación. }\end{array}$ & 2 \\
\hline $\begin{array}{l}\text { Is }=0.5 \text { Indica que el ecosistema se encuentra estático o en } \\
\text { transición, dependiendo de los agentes que interactúen con el } \\
\text { ecosistema en el futuro. }\end{array}$ & 1 \\
\hline $\begin{array}{l}\text { Is }<0.5 \text { y aproximándose más a 0, muestra que el ecosistema } \\
\text { sufre un severo impacto sinantrópico. }\end{array}$ & 0 \\
\hline $\begin{array}{l}\text { Is }=0 \text { Indica que la vegetación original está completamente } \\
\text { destruida. }\end{array}$ & 2 \\
\hline
\end{tabular}

Fuente: Ramón, Salinas y Lorenzo, 2013 
La combinación final entre la escala de naturalidad y el índice de sinantropismo se concreta sumando los valores asignados a las clases de sinantropismo (0-4) a los valores de la escala de naturalidad (1-10). De esta forma quedan clasificadas las unidades de paisaje bajo las siguientes clases (tabla 3):

Tabla 3. Clases de naturalidad y los rangos numéricos respectivos

\begin{tabular}{|c|c|l|}
\hline No. & Rangos & \multicolumn{1}{|c|}{ Estado de naturalidad } \\
\hline 10 & $\mathbf{1 4 - 1 3}$ & Sistema prístino \\
\hline 9 & $\mathbf{1 2 - 1 1}$ & Sistema natural \\
\hline 8 & $\mathbf{1 0}$ & Sistema subnatural \\
\hline 7 & $\mathbf{9}$ & Sistema cuasi natural \\
\hline 6 & $\mathbf{8}$ & Sistema semi natural \\
\hline 5 & $\mathbf{7}$ & Sistema cultural autosostenido \\
\hline 4 & $\mathbf{6}$ & Sistema cultural asistido \\
\hline 3 & $\mathbf{5}$ & Sistema muy intervenido \\
\hline 2 & $\mathbf{4 - 3}$ & Sistema semitransformado \\
\hline 1 & $\mathbf{2 - 1}$ & Sistema muy transformado \\
\hline
\end{tabular}

Fuente: Elaborado a partir de los valores obtenidos de la Escala de naturalidad y el Índice de sinantropismo

\section{Resultados y discusión}

Como primer paso se procedió a la confección del mapa de paisajes de la Reserva de la biosfera Buenavista (figura 2). Fueron identificadas ocho localidades y 62 comarcas en el área de estudio, evidenciando su alta diversidad físico-geográfica.

La localidad I está constituida por una llanura costera y cayos muy bajos que no sobrepasan los $2 \mathrm{~m}$ de altura y se divide en seis comarcas, atendiendo fundamentalmente al factor diferenciador edafo-biótico. Las localidades II y III constituyen una llanura baja, formada por 11 comarcas, y una llanura media, dividida en nueve comarcas, respectivamente. Por otra parte, la localidad IV está compuesta por alturas cársico - abrasivas, mogotiformes, con presencia de lapies, nichos marinos y cavernas, con alturas de entre 1-30 m; esta localidad se subdivide en cinco comarcas.

Constituida por una superficie de transición de 80-120 m de altura, se extiende en una estrecha franja hacia el sur del área de estudio la localidad 
Figura 2. Paisajes de la Reserva de la biosfera Buenavista

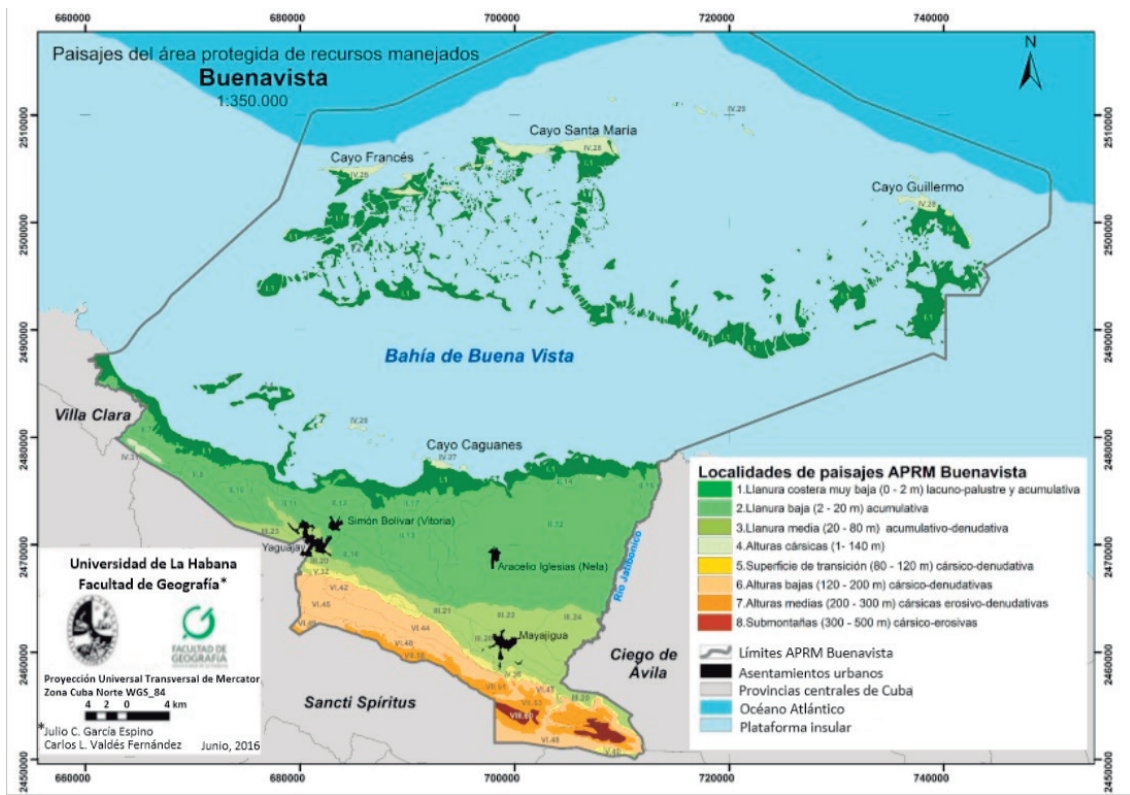

Fuente: Elaborado a partir del inventario de las unidades de paisaje del área de estudio

$\mathbf{V}$, esta precede a las alturas y submontañas y la componen 10 comarcas. La localidad VI consiste en una serie de alturas bajas cársico-denudativas, de entre 120-200 m de altura, mientras que la localidad VII está representada por alturas medias de origen cársico y erosivo-denudativo, de 200-300 m de altura; ambas localidades se subdividen en nueve y ocho comarcas, respectivamente. Por último, siguiendo en transición altitudinal a las alturas bajas y medias, prosigue la localidad VIII, la cual está representada por submontañas cársico-erosivas, de 300-500 m de altura, estas submontañas están representadas en una reducida superficie con componentes físicos homogéneos, lo que explica su subdivisión en cuatro comarcas.

Respecto a la estabilidad natural, la mayor proporción de las unidades de paisaje son estables (48,3\%), mientras que sólo el 14,5\% de las mismas constituyen unidades de paisaje inestables (figura 3 ). 
Figura 3. Mapa de la estabilidad natural y tecnogénica

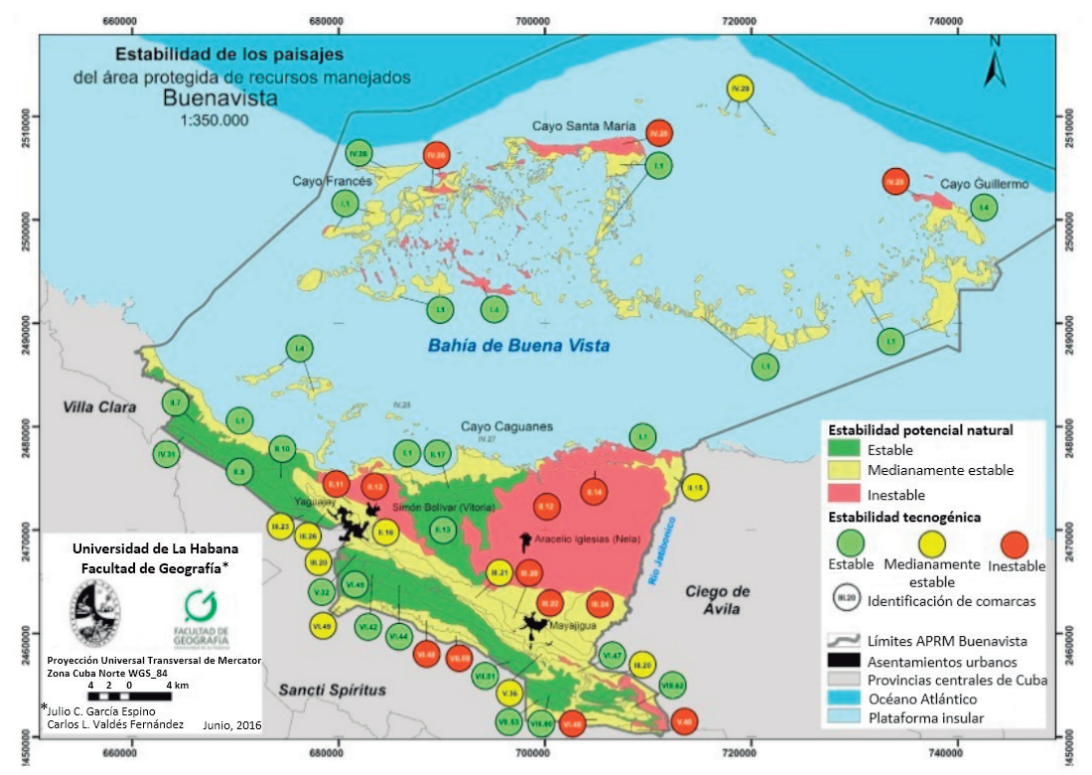

Fuente: Elaborado a partir del cálculo de la estabilidad natural y tecnogénica

Existe un predominio de las unidades estables y medianamente estables. La mayor cantidad de comarcas estables se distribuyen en las alturas medias, la llanura baja y alta y las alturas bajas. El mayor número de comarcas inestables pertenecen a la superficie de transición y a las alturas cársicas. Las llanuras muy baja y baja, las alturas bajas y las submontañas solo poseen una comarca inestable. Estas representan el 27,8 \% del área de estudio.

En cuanto a los factores de peligro externos evaluados, la incidencia de huracanes y tormentas tropicales, además de los sismos, no constituyen fenómenos de gran impacto sobre la estabilidad natural, pues presentan una baja incidencia sobre las distintas unidades de paisaje del área de estudio. Los resultados muestran que para el período de 1954-2014, solo afectaron directamente el área de estudio dos huracanes, de categorías uno y dos, respectivamente; mientras que la totalidad del área de la reserva posee un riesgo sísmico muy bajo.

Sin embargo, no sucede así en el caso de las sequías. La mayoría de las unidades se encuentran en peligro de sequía agrícola y meteorológica 
severo y moderado, las unidades en las que este factor influye con más fuerza se localizan hacia el norte del área protegida, en las llanuras bajas y altas. En el caso de las inundaciones, sobresalen las unidades con una susceptibilidad media y alta ante inundaciones por fuertes lluvias, las cuales se distribuyen, de igual modo, hacia la llanura costera y los cayos bajos, así como en la llanura baja.

Los factores de peligro internos muestran un comportamiento más heterogéneo en el área de estudio. En cuanto al grado de compactación del sustrato rocoso, se evidencia una clara diferenciación entre las unidades ubicadas al norte del área de estudio y las del sur, pues estas últimas están constituidas por rocas más antiguas y resistentes, mientras que las unidades ubicadas hacia el norte están compuestas por rocas menos resistentes a la compresión, como sedimentos marinos y palustres, areniscas y arcillas poco consolidadas. Algo similar ocurre con el grado de inclinación de la pendiente, pues en las llanuras, alcanza valores considerados como favorables, según su incidencia en la estabilidad natural, lo que contrasta con la superficie de transición, las alturas y submontañas del sur del área, además de las alturas cársicas.

En el caso de la capacidad protectora de la cubierta vegetal, se encuentran en un estado favorable las localidades donde se concentran los restos de bosque semideciduo en las alturas bajas, medias y las submontañas, además de los bosques micrófilos costeros y la vegetación de mangle que aparecen en la llanura costera muy baja y las alturas cársicas. Por otro lado, en las llanuras medias y bajas, la capacidad protectora de la cubierta vegetal se encuentra en un estado de desfavorable a medio, pues en estas se concentran las zonas de pastos y cultivos y los matorrales secundarios que han ido colonizando las áreas abandonadas por la actividad agrícola.

Por último, la erosión potencial, al tomar en cuenta tanto el grado de inclinación de la pendiente, como la cobertura vegetal, resume lo anteriormente expuesto, siendo la superficie de transición la que muestra las condiciones más desfavorables respecto a este factor, mientras de que en las alturas y submontañas, la cobertura vegetal regula el efecto negativo que ejercen las fuertes pendientes, del mismo modo en que, hacia las llanuras, la modificación de la vegetación original con fines agrícolas afectó de forma negativa la capacidad de erosión que presentan estas áreas, a pesar de contar con pendientes inferiores a $\operatorname{los} 6^{\circ}$. 
Respecto a la estabilidad tecnogénica se evidencia un predominio de las unidades estables. Las unidades inestables se distribuyen en las llanuras baja y alta, así como en la superficie de transición, ocupando más del $34 \%$ de la reserva. Estas áreas se caracterizan por presentar una fuerte modificación antrópica, con grandes extensiones de tierras ocupadas por pastos, cultivos y matorrales secundarios, además por la presencia de zonas urbanas; tal es el caso de las unidades antes señaladas.

Sin embargo, es válido señalar el caso de las unidades que coinciden con los cayos Santa María, Guillermo y Francés, pues las modificaciones antrópicas en estos, si bien en términos de área, no son relativamente tan extensivas, situación debido a la cual estas se incluyen en la clase medianamente estable; es necesario hacer llamar la atención sobre el control de estas áreas que se explotan desde hace unas décadas para el turismo. El desarrollo de la actividad turística en estas zonas plantea un conflicto en cuanto a la conservación de estas unidades, dado sus altos valores naturales, entre los que destaca el alto endemismo de su biota. De manera que, el hecho de que estas se incluyan como medianamente estables en cuanto a la estabilidad tecnogénica, no significa, necesariamente, que su situación con respecto a las modificaciones de origen antrópico sea favorable.

Al analizar de manera conjunta la estabilidad potencial natural y la estabilidad tecnogénica (figura 3 ) es evidente que todas las unidades de paisaje son susceptibles a la acción, tanto de factores de inestabilidad natural como de origen antrópico, sin embargo, no todas lo son en igual medida e intensidad. De forma general se muestra un patrón en el que coexisten unidades naturalmente inestables con las inestables y medianamente estables desde el punto de vista de la estabilidad tecnogénica.

De modo que, partiendo de la relación entre la estabilidad natural y tecnogénica, se caracterizó el comportamiento de la sensibilidad que presentan las diferentes unidades de paisajes en el área de estudio. En cuanto al área que ocupan las diferentes clases de sensibilidad, se observa que es muy similar el porcentaje que representan las unidades muy sensibles respecto a las muy poco sensibles (figura 4), por lo que, si bien, en cuanto a cantidad numérica de unidades muy sensibles y sensibles, estas no son significativas, sí lo son en cuanto a la extensión territorial que estas abarcan. 
Figura 4. Porcentaje de comarcas por clases de sensibilidad y el área que ocupan

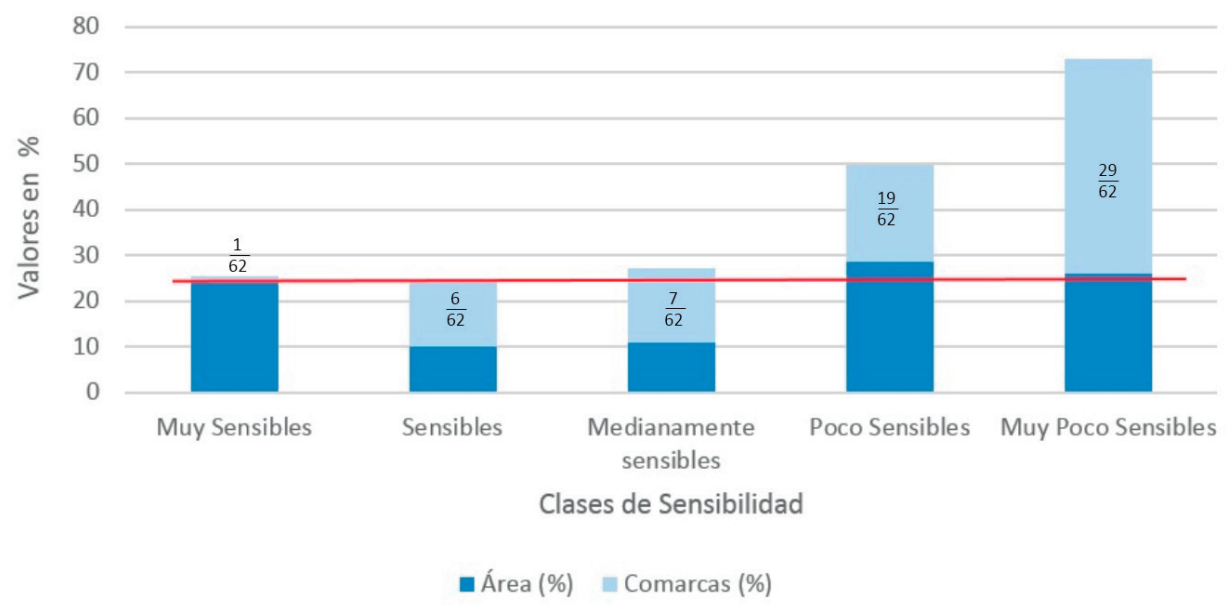

Fuente: Elaborado a partir del resultado de la sensibilidad de los paisajes

Según los resultados obtenidos, predominan las comarcas muy poco sensibles y poco sensibles. Las clases muy sensible y sensible se distribuyen en la llanura baja y alta, en la mayor parte de la superficie de transición y en las alturas cársicas al norte (cayos Santa María, Guillermo y Francés) (figura 5). En este grupo de unidades es donde se concentran las modificaciones de origen antrópico y, además, donde existen condiciones naturales que las hacen más susceptibles ante los factores de inestabilidad natural. Por tanto, estas constituyen las unidades más frágiles en cuanto a su capacidad de adaptación o recuperación ante los efectos modificadores debidos a la acción de factores externos e internos, ya sean de origen natural o antrópicos.

A pesar de que solo 10 unidades del total son muy sensibles o sensibles, estas ocupan un área considerable de la reserva, sobre todo en las llanuras baja y alta. Esto se debe al hecho de que, la actividad humana se ha concentrado en las comarcas que a su vez son las de mayor extensión, a lo que se añaden las condiciones desfavorables que estas presentan en cuanto a su comportamiento ante los factores naturales de peligro interno y externos. 
Figura 5. Mapa de la sensibilidad de los paisajes

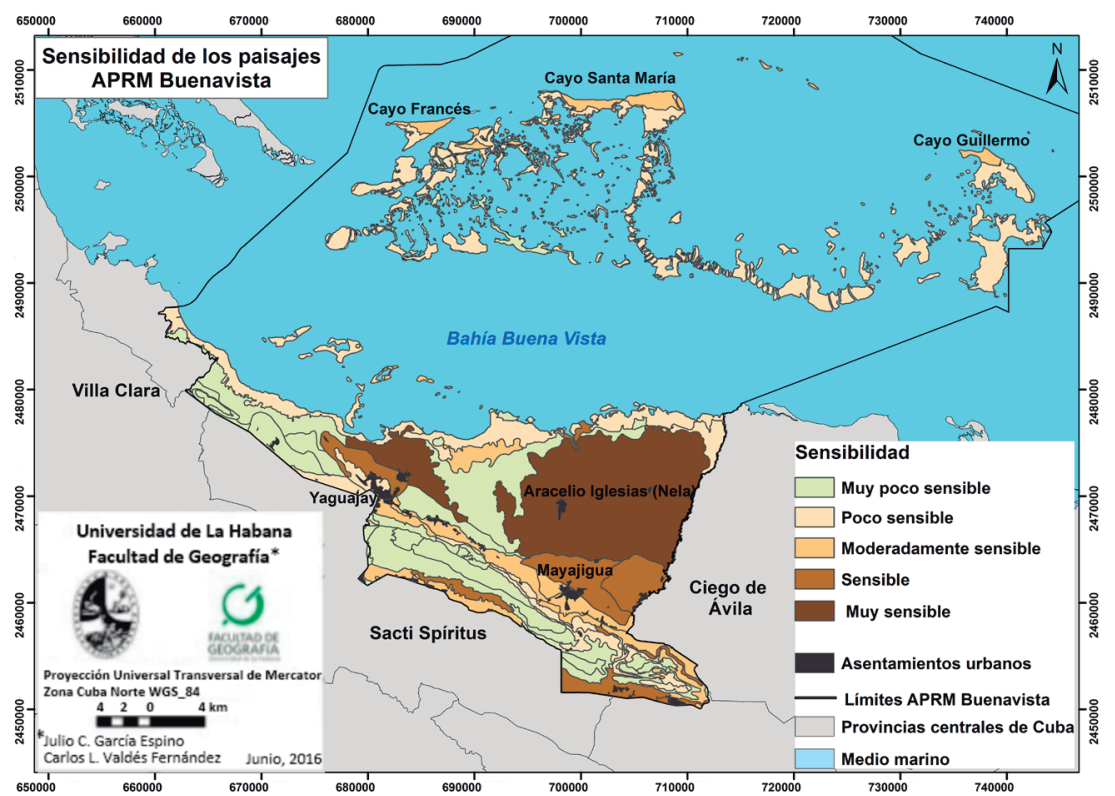

Fuente: Elaborado a partir del resultado de la sensibilidad de los paisajes

Las unidades sensibles en las llanuras alta y baja evidencian en sus condiciones de estabilidad y sensibilidad el proceso de ocupación que estos han sufrido a partir del aprovechamiento de sus recursos por el hombre, el cual data de los tiempos coloniales. Sin embargo, en el caso de las unidades de los cayos, su aprovechamiento data de finales del siglo pasado y viene dado por su explotación para el turismo, actividad que ha de ser monitoreada y estudiada en el área con el fin de eliminar, reducir y prevenir los efectos negativos de la misma sobre estas unidades sensibles.

\section{Naturalidad}

La naturalidad del área es un indicador resultante del análisis conjunto de la escala de naturalidad y el índice de sinantropismo.

La escala de naturalidad, con un basamento subjetivo, valoró entre los criterios diagnósticos negativos, el elemento biótico exótico dado por las especies invasoras, sobre todo Dichrostachys cinerea (marabú) y Acacia farnesiana (aroma) en la zona de las localidades de la llanura baja 
y media, con tendencia al aumento hacia la base de la superficie de transición. Esto responde a la relación bien comprobada de la ganadería y la expansión de estas especies, dado por el tipo de diseminación pasiva por zoocoría que ejerce el ganado. También la Oeceoclades maculata, (orquídea de tierra o africana) invade la superficie de transición, registrándose cada vez más individuos en esas comarcas.

El criterio diagnóstico elementos artificiales, en cuanto a los contaminantes, fueron calificados de permanentes en el agua, en las unidades de paisajes donde se encuentran los asentamientos de Yaguajay y Mayajigua, principalmente, y en aquellas unidades de la llanura baja donde se localizan los asentamientos de Simón Bolívar (conocido también por su antiguo nombre Vitoria) y Aracelio Iglesias (Nela), y en Obdulio Morales (Narcisa). Esta contaminación es principalmente orgánica, proveniente de las actividades domésticas y la cría de porcinos. Este último es mucho mayor en los últimos asentamientos mencionados, los cuales cambiaron de actividad económica principal a inicios de este siglo: de la producción de azúcar a la actividad agrícola. Estos residuales líquidos se vierten a la red de drenaje superficial, que desemboca generalmente en la zona costera, principalmente en el manglar.

Dentro de los elementos artificiales, también se incluyen las instalaciones, que son dominantes en las unidades paisajísticas que incluyen los asentamientos. Otras unidades en las que se categoriza como claramente dominantes son los cayos donde se han construido numerosas infraestructuras pertenecientes a la actividad turística (hoteles, aeropuertos, viales).

Para la entrada de energía y materia se tuvo en cuenta dos fuentes: natural y de origen antrópico. La primera fuente considera el relieve, la red hidrográfica, la dinámica costera, entre otros; la segunda el flujo poblacional, de transporte, la entrada y salida de insumos y personas, de electricidad, de agua, etc. Las unidades valoradas con menor entrada fueron aquellas más alejadas de las alturas como los cayos bajos, en los que no se recibe aporte de sedimentos provenientes de zonas altas, así como tampoco energía o materia de origen antrópico debido a la inaccesibilidad. También las unidades de paisajes más altas y puntuales fueron calificadas como de baja entrada, debido a que son áreas emisoras y dónde la actividad humana es escasa. Las comarcas con mayor entrada son aquellas receptoras de energía y/o materia proveniente de las unidades de alturas 
como las llanuras medias y bajas, así como los cayos donde se desarrolla el turismo intensivo. En ambos espacios, el ingreso debido a las actividades socioeconómicas es elevado.

Al analizar el criterio de alteraciones físicas, se reiteran las unidades con mejores condiciones y mayor disponibilidad de recursos de interés para las actividades humanas. Son las llanuras medias y bajas, así como los cayos elevados y cársicos con turismo de sol y playa, las unidades de paisajes con mayor cantidad de viales, edificaciones, modificación de la topografía y remoción de suelos, etc. Hacia el lado opuesto, las localidades y comarcas con menos alteraciones físicas son aquellas poco explotadas como las alturas, las costeras bajas, así como los cayos de mangles.

La extracción de elementos es un criterio que tiene en cuenta el consumo de recursos como el agua, minerales, especies de la biota, etc. En el caso de la superficie de transición, sobre todo en aquellas localidades cercanas a los asentamientos poblacionales, existe de forma puntual la extracción de agua producto de la utilización de los manantiales identificados con fines de consumo humano. También la roca caliza, es extraída en zonas de pendientes agudas, constituyendo casos de minería intensiva de manera puntual. En las unidades de las alturas se evidencia la tala selectiva de especies maderables y el desbroce puntual para la ganadería doméstica. Algunas especies animales, principalmente aves, son también extraídas de su hábitat.

En la llanura media y baja, la extracción de aguas subterráneas también es intensiva, llegando a ser identificada como peligrosa en cuanto a la depresión crítica del manto freático, con efectos serios en la disponibilidad para las actividades domésticas y la consecuente penetración de la cuña salina. Si se analiza la extracción de elementos, a partir de un estadio primigenio, son aquellas unidades que albergan las áreas socioeconómicas, las que han registrado una mayor extracción histórica. Por esto, se consideran la llanura media y baja, las de mayores niveles de extracción.

La fragmentación es tratada teniendo en cuenta la continuidad espacial dentro de las unidades de paisajes. Las evidencias son aquellos espacios cuya cobertura original o secundaria no existe, y en su lugar se encuentra expresada una fisionomía producto de alguna actividad antrópica o restos de ella. Los paisajes más fragmentados son las localidades de la llanura media y alta. Estas unidades incluyen las principales áreas de 
cultivos, asentamientos humanos e infraestructuras relacionadas, evitando la continuidad de la cobertura vegetal de norte a sur, desde los manglares y el bosque siempreverde de ciénaga hasta los bosques semideciduos mesófilos de las alturas.

También algunas unidades tienen fragmentación evidente como los cayos altos y cársicos, donde se asienta el turismo, cuyas instalaciones han subdividido el matorral xeromorfo costero, así como algunas áreas de manglares y saladares interiores. También el sistema de alturas, tiene parches con usos que cambian la cobertura original, impidiendo una continuidad compacta de este a oeste. Sobre todo la superficie de transición, donde la ganadería ha penetrado. Las unidades cuya cobertura no ha sido fraccionada se localizan en las alturas y submontañas, así como la línea de mangles inundada, los cayos bajos y algunas comarcas de la llanura baja. Se destaca la comarca II.13, muy relacionada espacialmente con la ciénaga de la Guayabera. Al ser una zona baja e inundable, la agricultura no colonizó totalmente esta zona, que al ver disminuido la intensidad de las actividades agrícolas en las últimas décadas, ha comenzado una sucesión ecológica secundaria.

El último criterio de evaluación es la dinámica, entendido como el nivel de funcionamiento de los elementos más importantes como la radiación, el agua, la biota. Varía en dependencia de la manipulación, control y dominio que el hombre ejerce sobre estos elementos. De esta manera, niveles de contaminación atmosférica o modificaciones del relieve, o eliminación de la cobertura boscosa, modifican los valores de la incidencia de radiación en superficie. El represamiento, la canalización del escurrimiento superficial o el desvío de ríos también alteran la dinámica natural; y cuando se transforman los hábitats, se introducen especies y se crean barreras, lo que impide las relaciones y flujo de los organismos. Teniendo en cuenta todo esto, los paisajes con la dinámica más alterada son los presentes en la llanura media y alta, así como en los cayos cársicos con un uso turístico. La superficie de transición y algunas unidades de las alturas, aunque en menor medida, también tienen una dinámica modificada. Aquellas unidades, cuya estructura y funcionamiento han permanecido estable, son las mayores alturas, sectores de la línea baja costera y los cayos que no han sido explotados. 
En cuanto al cálculo del índice de sinantropismo, como segunda parte del indicador naturalidad, el primer paso consistió en clasificar cada especie registrada dentro del área según su categoría sinantrópica, como fue referido antes en la metodología.

Los paisajes con mayor impacto sinantrópico, entiéndase las áreas con mayor proporción de especies antropófitas, o sea, con una biota más transformada, son las llanuras baja y media. En menor medida los cayos Santa María, Las Brujas y Guillermo. También las unidades de las alturas fueron clasificadas como ecosistemas estáticos o en transición. Se decide alertar sobre la superficie de transición, debido a los reportes muy recientes provenientes de especialistas en el área, de presencia de especies invasoras y por su cercanía a áreas con alta manipulación debido a actividades agrícolas.

Figura 6. Indicador naturalidad en la Reserva de la biosfera Buenavista

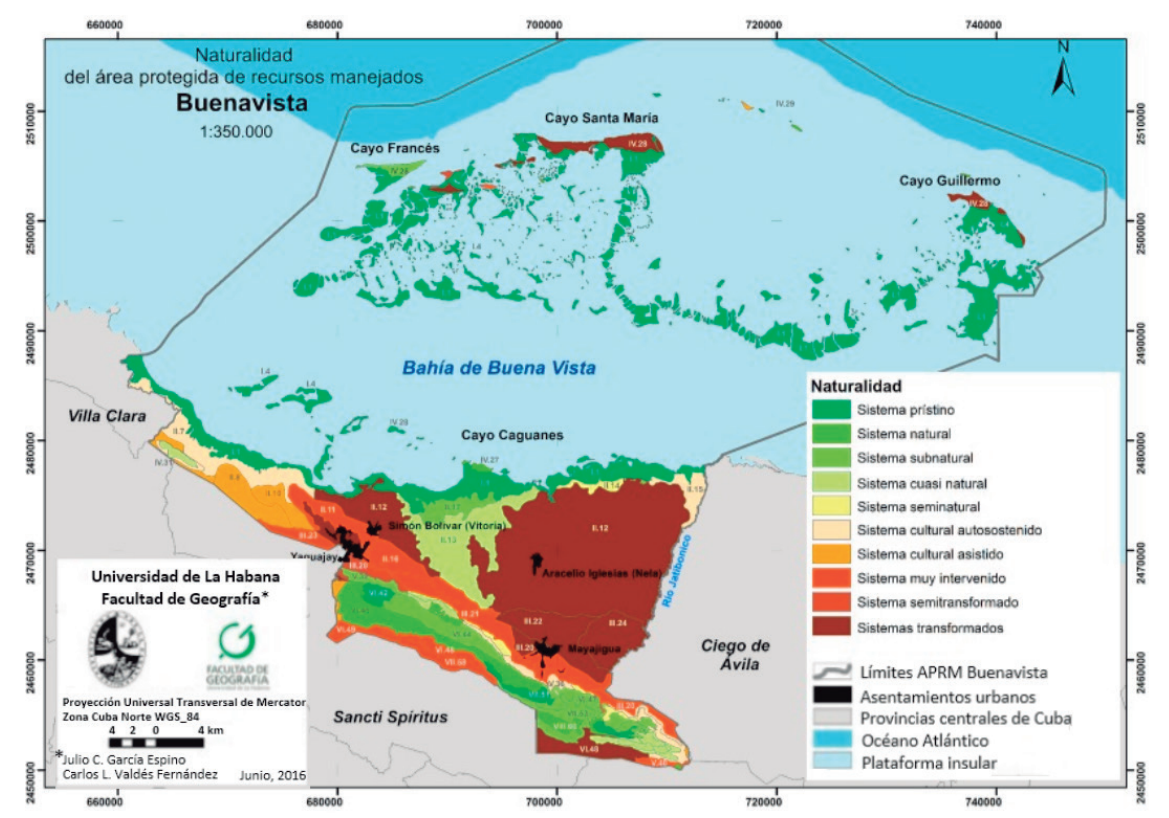

Fuente: Elaborado a partir del cálculo del indicador naturalidad

La combinación final entre la escala de naturalidad y el índice de sinantropismo (figura 6) evidenció que los sistemas prístinos (zonas vírgenes) toman área en las unidades de paisaje de muy difícil acceso, 
principalmente los bosques de mangles en los cayos, los bosques semideciduos mesófilos sobre las alturas y el complejo de vegetación de mogote.

Los sectores del APRM con características de zonas vírgenes corresponden a la llanura muy baja, los cayos y la franja litoral de manglar. También la comarca de las alturas bajas contenida en el área protegida Jobo Rosado, así como las comarcas de las alturas medias con cobertura de bosques semideciduos conservados y complejo de vegetación de mogote (al este), se clasifican como sistemas prístinos. Las zonas incluidas dentro de esta clasificación se caracterizan por el predominio casi total de elementos y procesos naturales, solo con presencia absolutamente insignificante de elementos antrópicos, o totalmente despreciable de polución físico-química de fuentes antrópicas exteriores. Esta situación es entendible debido a que son áreas muy inaccesibles donde la acción del hombre ha sido exploratoria.

Así también, las áreas más beneficiosas para el desarrollo socioeconómico son las que se encuentran en las categorías de sistemas transformados, como varias comarcas de las llanuras baja y media y los cayos Santa María y Guillermo de las alturas cársicas. Estas áreas han sido asimiladas de forma intensiva por el hombre, convirtiéndolas en espacios socioproductivos, en los cuales se desarrollan varias actividades económicas como la agricultura, la ganadería y el turismo. En estas comarcas dominan los procesos antrópicos, con una clara preponderancia de elementos artificiales; los elementos naturales son exóticos, decorativos o no visibles y existe una total dependencia de entradas de materia y energía desde el exterior.

En general, la naturalidad baja, representada por los sistemas intervenidos y alterados, donde es común la presencia de construcciones e infraestructuras, así como la existencia de una diversidad natural severamente reducida, con elementos naturales intensamente fragmentados y modificados, es mayor y más concentrada (en ocupación de área) que la naturalidad alta; representada por los sistemas prístinos y naturales, donde la presencia de elementos artificiales es escasa y dispersa y la polución está ausente o es capaz de ser asimilada por el sistema.

\section{Conclusiones}

Al concluir la cartografía de los paisajes de la Reserva de la biosfera Buenavista, como base para el estudio de los mismos, se delimitaron ocho 
localidades y 62 comarcas. Tres de ellas referidas a llanuras, tres a sistema de alturas, una al relieve cársico de alturas aisladas y otra a una superficie de transición entre las llanuras y el sistema de alturas. Los principales factores geoecológicos causantes de la diferenciación fueron en primera instancia el relieve, y después el suelo, la cobertura vegetal y el uso.

Para el análisis de las distintas unidades de paisaje se desarrollaron tres indicadores. Entre ellos, en la determinación de los distintos grados de estabilidad se observa un predominio de las unidades con estabilidad potencial natural alta (estables). Las comarcas inestables representan el $28 \%$ de la superficie del área de estudio, entre las que se destacan la superficie de transición y las alturas cársicas. Se evidencia que, el predominio de las unidades con mayor estabilidad natural corresponden a las comarcas de la llanura baja con cobertura vegetal no cultural y las localidades de alturas, mientras que las menos estables se corresponde con aquellas donde la cobertura vegetal es nula y que son susceptibles ante factores de peligro como inundaciones y sequías.

La estabilidad tecnogénica muestra un patrón similar al de la natural, observándose el predominio de las unidades estables. Existe coincidencia regular con la estabilidad natural, pues la variable que se toma en cuenta para determinar la estabilidad tecnogénica es la proporción del área de la unidad de paisaje que se encuentra antropizada. La transformación de estos espacios aumenta la vulnerabilidad de estas zonas ante los distintos factores de peligro interno y externos, fundamentalmente, la capacidad protectora de la cobertura vegetal, la erosión potencial y la incidencia de sequías e inundaciones.

Al analizar el comportamiento conjunto de la estabilidad natural y la tecnogénica se determinaron los distintos grados de sensibilidad de las unidades en el área. Se evidencia el predominio de las unidades muy poco sensibles y poco sensibles, mientras que las unidades sensibles y muy sensibles coinciden con las de mayor extensión dentro del área, destacándose las llanuras baja y alta, las cuales requieren de una mayor vigilancia y control de las actividades humanas, con vistas a minimizar los impactos negativos sobre las mismas.

Según la naturalidad, que incluyó en su confección el análisis conjunto de la escala de naturalidad y del índice de sinantropismo, las unidades de paisajes clasificadas como sistemas poco alterados coinciden con 
las localidades poco accesibles y desventajosas para las actividades socioeconómicas, como es el caso de la llanura costera muy baja, ocupada por manglares y herbazal de ciénaga y las alturas. Por otro lado, aquellas localidades con condiciones favorables para el desarrollo de la agricultura, el turismo, la industria y el enclave de asentamientos humanos (llanura baja y media, y las alturas cársicas como las zonas elevadas de los cayos Santa María y Guillermo) constituyen sistemas transformados, con una alta modificación de su cobertura vegetal y un predominio evidente de los procesos antrópicos.

\section{Referencias bibliográficas}

Acevedo, P. (2014). Fundamentos Geográficos del Medio Ambiente. La Habana, edit. Félix Varela, Tomo I.

Acevedo, P. R. (1996). Análisis de los paisajes insulares del archipiélago Sabana-Camagüey, Cuba. (Tesis de Doctorado, Facultad de Geografía), Universidad de La Habana.

Agencia de Medio Ambiente (AMA) (2012). Estudios de Peligro, Vulnerabilidad y Riesgos de la provincia Ciego de Ávila. Afectaciones por fuertes vientos, inundaciones por intensas lluvias e inundaciones por penetración del mar. Informe Académico. Grupo Técnico de Estudios de PVR, provincia Ciego de Ávila, Cuba.

Agencia de Medio Ambiente (AMA) (2012). Estudios de Peligro, Vulnerabilidad y Riesgos de la provincia Sancti Spíritus. Afectaciones por fuertes vientos, inundaciones por intensas lluvias e inundaciones por penetración del mar. Informe Académico. Grupo Técnico de Estudios de PVR, provincia Sancti Spíritus, Cuba.

Agencia de Medio Ambiente (AMA) (2012). Estudios de Peligro, Vulnerabilidad y Riesgos de la provincia Villa Clara. Afectaciones por fuertes vientos, inundaciones por intensas lluvias e inundaciones por penetración del mar. Informe Académico. Grupo Técnico de Estudios de PVR, provincia Villa Clara, Cuba.

Aguilar, K. (2009). Análisis geoecológico de los paisajes de la Reserva de la Biosfera Sierra del Rosario. (Trabajo de Diploma, Facultad de Geografía), Universidad de La Habana.

Burgui, L. B. (2010). Impactos ambientales de la ocupación turística sobre la sostenibilidad de los paisajes naturales de Cayo Santa María 
(Villa Clara, Cuba). (Tesis de Maestría). Universidad de La Habana, La Habana, Cuba.

Calvo, D. (2013). Diagnóstico geoecológico de los paisajes de la reserva ecológica "Lomas de Banao. (Trabajo de Diploma, Facultad de Geografía), Universidad de La Habana.

Centro Nacional de Huracanes (HNC, siglas en inglés). (2014): Bases de datos de huracanes en el caribe (1815-2014). Recuperado de http:// www.nhc.noaa.gov. 23/05/2016.

Chuy, T. J. (2001). Macrosísmica de Cuba y su aplicación en los estimados de peligrosidad sísmica. IV Congreso de Geología y Minería Geofísica, La Habana.

Cruañas, E. (2013). Diagnóstico geoecológico de la cuenca Ariguanabo, provincia Artemisa. (Trabajo de Diploma, Facultad de Geografía), Universidad de La Habana.

Estrada, E. R. et al. (2013). Mapa (BD-SIG) de vegetación natural y seminatural de Cuba V.1, sobre Landsat ETM-7 SLC-OFF GAP FILLED, CIRCA 2011. VIII Congreso Internacional de Geomática, La Habana.

Fajardo, G. (2008). Diagnóstico geoecológico del paisaje natural protegido Guajaibón como premisa para su plan de plan de manejo integral. (Trabajo de Diploma, Facultad de Geografía), Universidad de La Habana.

García, A. (2002). Análisis de los paisajes y ordenamiento geoecológico del Parque Nacional Caguanes. (Trabajo de Diploma, Facultad de Geografía), Universidad de La Habana.

Lemes, G.M. (2000). Diagnóstico geoecológico de los paisajes del Sector Guantánamo de la Empresa Agroforestal de Montaña "General Antonio Lince”. (Tesis de Maestría, Facultad de Geografía), Universidad de La Habana.

Mateo, J. M. (2000). Geografía de los Paisajes. Facultad de Geografía, Universidad de La Habana.

Mateo, J. M. (2011). Geografía de los Paisajes. Paisajes Naturales. La Habana, edit. Félix Varela, Tomo I.

Organización de las Naciones Unidas para la Educación, la Ciencia y la Cultura (UNESCO) (2015). Estrategia del programa El Hombre y la Biosfera para el periodo 2015-2025. París. 
Ramón, P. A. M., Salinas C. E. y Lorenzo, M. C. (2013). Guía metodológica para la zonificación de áreas protegidas terrestres de Cuba. (Inédito)

Ricardo, N. E., Pouyú, E. y Herrera, P.P. (1990). Clasificación de la flora sinantrópica de Cuba, Revista del Jardín Botánico Nacional, 11(23), 129-133.

Sánchez, R. (2005). Diagnóstico geoecológico de los paisajes del parque nacional Viñales. (Trabajo de Diploma, Facultad de Geografía), Universidad de La Habana. Cuba.

Sigarreta, S. (2000). Diagnóstico geoecológico del municipio Holguín. (Tesis de Maestría, Facultad de Geografía), Universidad de La Habana. Cuba.

Venegas, C. (2004). Diagnóstico geoecológico de la reserva ecológica "Limones-Tuabaquey". (Tesis de Maestría, Facultad de Geografía), Universidad de La Habana. Cuba. 